\title{
Can Halogen Clusters be Emissive?
}

Zihao Zhao, Saixing Tang, Siyu Tao, Tianjia Yang, Yueying Lai, and Wang Zhang Yuan*

School of Chemistry and Chemical Engineering, Frontiers Science Center for Transformative Molecules, Shanghai Key Lab of Electrical Insulation and Thermal Aging, Shanghai Jiao Tong University, No. 800 Dongchuan Rd., Minhang District, Shanghai 200240, China

E-mail:wzhyuan@sjtu.edu.cn

\begin{abstract}
Halogen-halogen short contacts, especially halogen bonds (XBs) have been widely utilized in multifarious fields, owing to its bridging function among luminophores as well as well-known heavy atom effect. However, little attention has been paid to the luminescent ability of halogen clusters. It remains unknown whether they are emissive. Herein, inspirited by the clustering-triggered emission of nonconventional luminophores, we report the first examples of emissive halogen clusters with fluorescence-phosphorescence dual emission in aggregated state and even under ambient conditions. Additionally, multi-tunable PL in response to excitation wavelength, temperature, and pressure are noticed. These results shed new lights on the underlying emission mechanism and would inspirit further exploration of nonconventional luminophores involving halogen moieties.
\end{abstract}

Keywords: clustering-triggered emission, halogen clusters, halogen-halogen short contacts, multitunable photoluminescence, nonconventional luminophores 
Halogen bond $(\mathrm{XB})$ is a kind of burgeoning electron donation-based noncovalent interaction between halogen atoms (XB donor) and electron-rich Lewis base (XB acceptor), ${ }^{1-3}$ thanks to the electrophilic portion termed as $\sigma$-hole on halogen atoms at the elongation direction of the $\mathrm{R}-\mathrm{X}$ covalent bond $(\mathrm{R}=\mathrm{C}$, N, halogen, etc.) (Figure 1A). ${ }^{4}$ Relating researches were just launched recently after decades of being shelved, ${ }^{5}$ wherein $\mathrm{XB}$ has participated in multitudinous fields like crystal engineering, ${ }^{6-8}$ molecular recognition, ${ }^{9,10}$ as well as supramolecular chemistry, ${ }^{11}$ etc. as an effective tool, owing to its moderate strength (5-180 kJ/mol) and superior directionality. ${ }^{12}$ In that scenario, XB holds broad applications serving as a universal noncovalent intermolecular interaction in molecular engineering.

In addition to broad applications included above, XB has also become an effective pathway in constructing pure organic luminogens, which are playing increasingly significant roles in cell imaging, ${ }^{13,14}$ optoelectronics, ${ }^{15-18}$ bio/chemo probes, ${ }^{19,20}$ and medical therapy. ${ }^{21,22}$ The advantages of XB introduction to photoluminescence (PL) are multifaceted. Not only can XB act as a mighty candidate to multiply intermolecular interactions, similar to other noncovalent interactions (e.g. hydrogen bond, chalcogen bond, ionic and dipole-dipole interactions), with the aim of promoting and regulating photophysical properties, ${ }^{23-25}$ also the heavy atom effect and ample lone pair (n) electrons of halogen atoms will create an enabling environment for spin-orbit coupling (SOC) and subsequent intersystem crossing (ISC) process, resulting in boosted triplet excitons. ${ }^{26,27}$ Therefore, XB is widely utilized in the construction of pure organic luminophores, particularly those with efficient room temperature phosphorescence. ${ }^{26-29}$ 


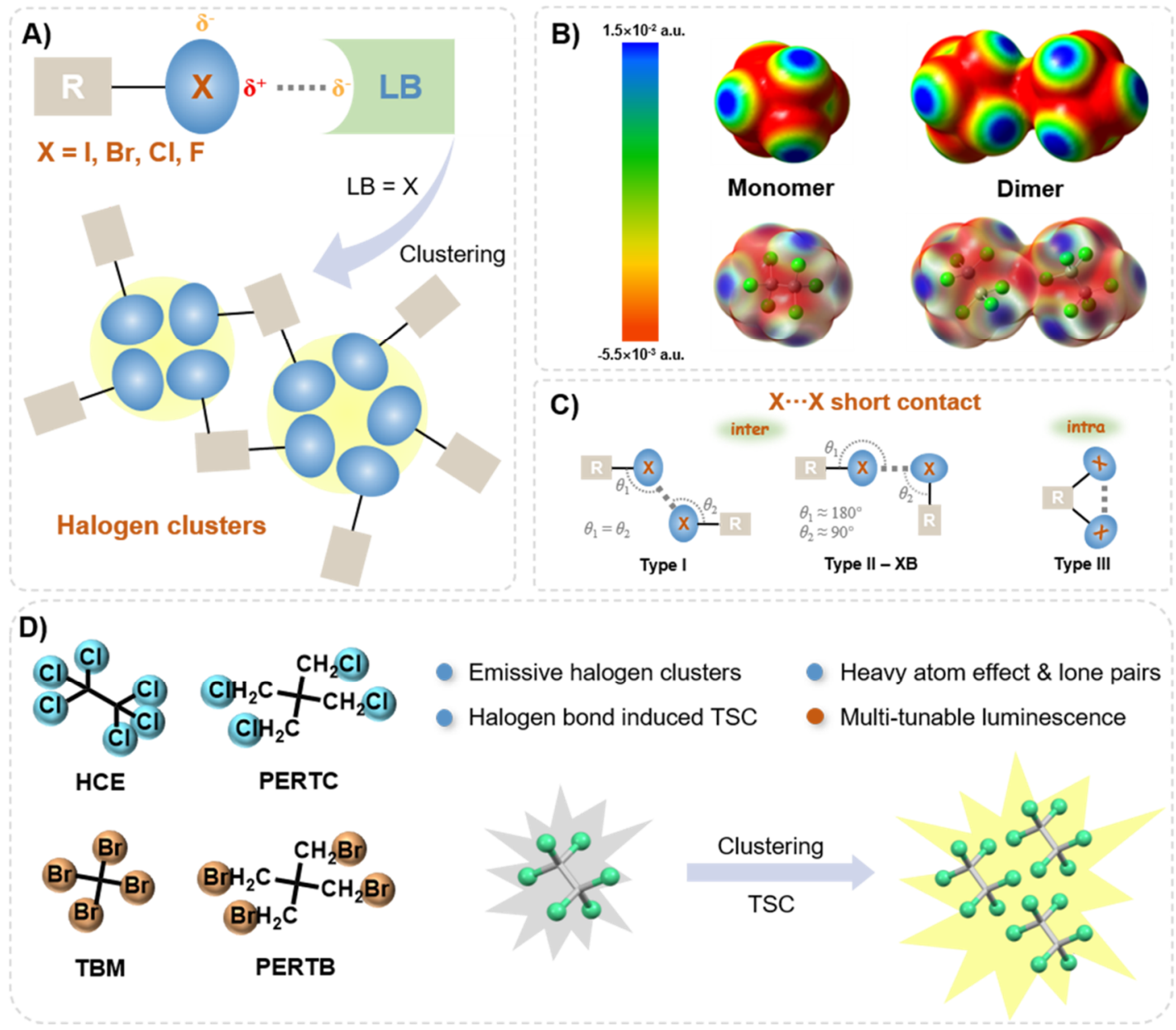

Figure 1 A) Schematic illustration of $\mathrm{XB}$ and halogen clusters (LB = Lewis base). B) MEP at the isodensity surface for HCE monomer and dimer. C) Structural scheme for inter- (type I and type II) and intramolecular (type III) halogen $\cdots$ halogen short contacts. $\mathrm{X}=$ halogen atom, and $\mathrm{R}=\mathrm{C}, \mathrm{N}, \mathrm{O}$, halogen atoms, etc. Note: Type II contacts are XBs. D) Structural of HCE, PERTC, PERTB, and TBM, and schematic illustration of the individual (nonemissive) and clustered (emissive) HCE molecules.

Thus far, however, to the best of our knowledge, $\mathrm{XB}$ or halogen atom in these systems only plays a supporting role in regulating PL emission, whereas no research concerning PL ability of pure halogen clusters (Figure 1A) was reported, presumably on account of lacking evident luminescent chromophores. Nevertheless, the molecular electrostatic potential (MEP) analysis of hexachloroethane (HCE) dimer (with an obvious $\mathrm{Cl} \cdots \mathrm{Cl} \mathrm{XB}$ ) illustrates a well shared electron cloud and expanded delocalization (Figure 1B). Its trimer and tetramer (Supporting Information, Figure S1) show similar extended electron 
delocalization, which is beneficial to emission. Moreover, besides XB demonstrated in Figure C (type II), simple intra- and intermolecular halogen-halogen short contacts without the involvement of electrophilic $\sigma$-hole (Figure 1C, type I and III) are believed to exist, ${ }^{2}$ which further facilitate electron communication between HCE molecules. Inspired by such calculated results and the intrinsic PL from various nonconventional luminophores with electron-rich moieties (bearing $\mathrm{n}$ and/or $\pi$ electrons), ${ }^{30-32}$ and regarding the electron-rich character of halogen atoms, herein, photophysical properties of some aliphatic haloalkanes adopting pure halogen clusters have been investigated for the first time. Considering fluoroalkane is poor XB donor ${ }^{12}$ while iodoalkanes are mostly chemically unstable ${ }^{33}$ four commercially available chloro- and bromoalkanes, namely HCE, pentaerythrityl tetrachloride (PERTC), pentaerythrityl tetrabromide (PERTB), and tetrabromomethane (TBM) (Figure 1D) were adopted as representatives. For these haloalkanes, diversified halogen clusters with efficient intra- and intermolecular through-space conjugation (TSC) $)^{34-38}$ and prominent heavy atom effect are expected. It is found that individual haloalkane molecules are difficult to excite and thus cannot demonstrate notable PL, while their aggregates are capable of emitting, owing to the formation of halogen clusters with effective TSC (Figure 1D). Their single crystals exhibit clearly visible PL and instrument-detectable room temperature phosphorescence (RTP) under 312 and 365 nm UV lights, accompanying evident excitation wavelength $\left(\lambda_{\mathrm{ex}}\right)$-dependent PL. Meanwhile, an eye-catching multi-tunability of these haloalkanes responding to $\lambda_{\mathrm{ex}}$, temperature, and pressure has also been observed.

As Figure 2A demonstrated, weak but visible PL from single crystals of these haloalkanes was noticed. With the variation of $\lambda_{\mathrm{ex}}, \mathrm{HCE}$ crystals, as the representative, show different PL intensities with maxima varying from UV-A to visible region at 365/376/434/443/451/530 nm (Figure 2B, upper), which should be ascribed to the coexistence of diverse emissive species. Further time-resolved measurement at ns scale discloses disparate lifetimes $\left(<\tau>_{\mathrm{f}}\right)$ of 2.1 and $2.7 \mathrm{~ns}$ at 429 and $454 \mathrm{~nm}$ (Figure 2C), respectively, thus confirming the concurrence of heterogeneous emissive species. Similar PL phenomena were also observed from PERTC, PERTB and TBM crystals (Figure S2, S3). These emission behaviors, analogous 
to most nonconventional luminophores, can be readily rationalized by the cluster-triggered emission (CTE) mechanism. More surprisingly, although couldn't be captured by naked eyes or digital camera due to the extreme weakness, persistent RTP (p-RTP) from these haloalkanes was still detected by spectrometer with $312 \mathrm{~nm}$ UV irradiation, as suggested by their lifetimes $\left(<\tau>_{\mathrm{p}}\right)$ of 58.4 and $30.9 \mathrm{~ms}$ at 529 and $605 \mathrm{~nm}$ for HCE crystals (Figure 2B, 2D and Figure S2, S4). Notably, the broad emission spectra and distinct $\left\langle\tau>_{\mathrm{p}}\right.$ values highly indicate the concurrence of multiple emissive populations.

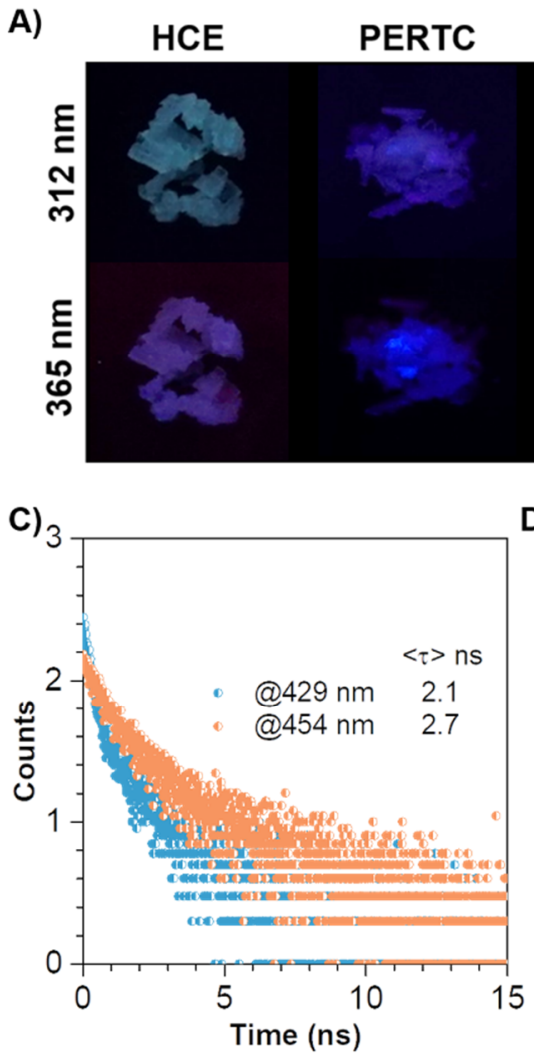

PERTB

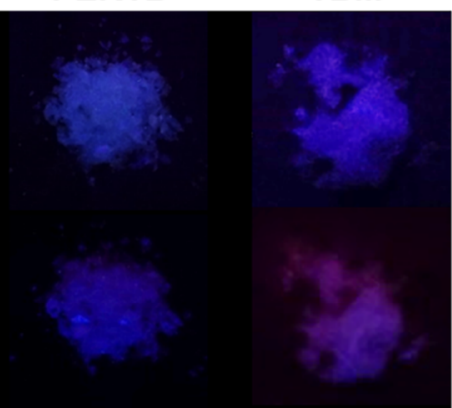

D) 3

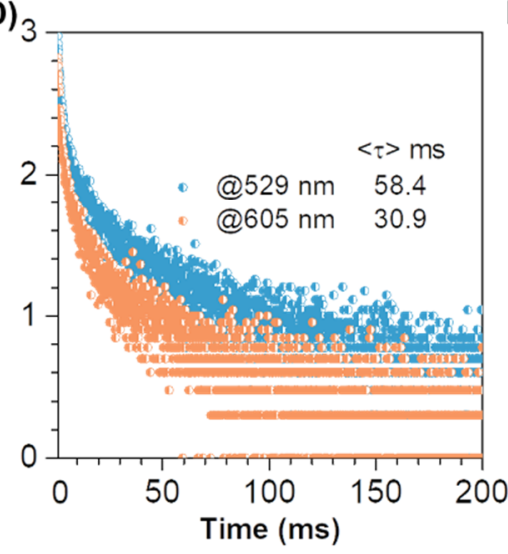

B)

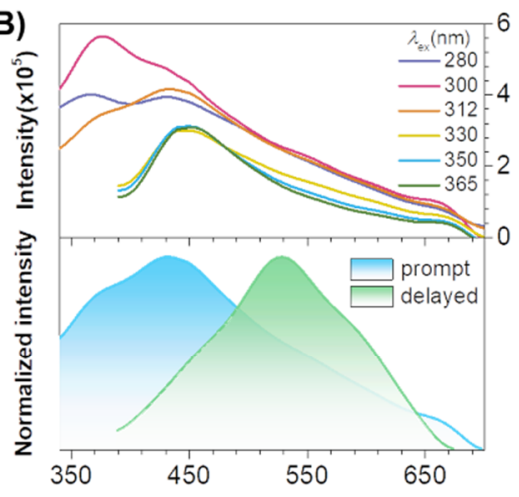

E)

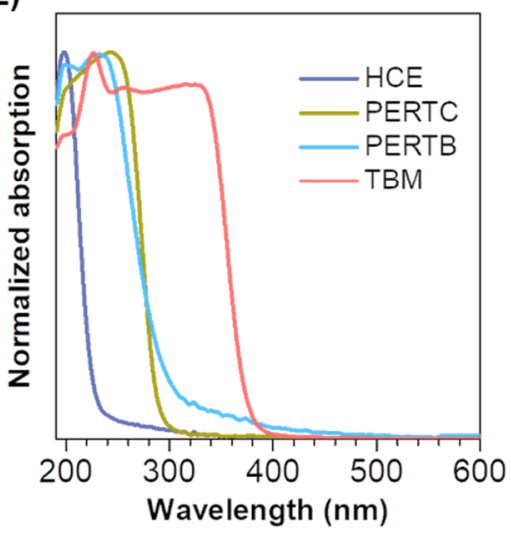

Figure 2. A) Luminescent photographs of HCE, PERTC, PERTB, and TBM single crystals taken under 312 and $365 \mathrm{~nm}$ UV lights. B) Prompt $\left(t_{\mathrm{d}}=0\right)$ emission spectra with different $\lambda_{\mathrm{ex}} \mathrm{S}$ (upper) as well as prompt $\left(t_{\mathrm{d}}=0\right)$ and delayed $\left(t_{\mathrm{d}}=0.1 \mathrm{~ms}\right)$ emission spectra with $312 \mathrm{~nm} \mathrm{UV}$ irradiation (lower) of HCE crystals. C) Nanosecond (ns, $\lambda_{\mathrm{ex}}=363 \mathrm{~nm}$ ) and D) millisecond ( $\mathrm{ms}, \lambda_{\mathrm{ex}}=312 \mathrm{~nm}$ ) scale lifetimes for HCE crystals monitored at different $\lambda_{\mathrm{em}} \mathrm{s}$. E) Absorption spectra of HCE, PERTC, PERTB, and TBM single crystals. 
Furthermore, diversity of halogen clusters can also be testified by extensive absorption containing multiple peaks in UV-vis spectra (Figure 2E), wherein obvious bands attributed to XBs due to charge transfer transitions can be found arising in the UV region ${ }^{5}$. Additionally, such wide absorption region from UV-A to near visible region (especially the one for TBM) gives rational interpretation to the behavior that these samples are ready to be excited by even UV-A irradiation. Apart from the diversity of emissive species at the ground state testified by UV-vis spectra, the heterogeneous popularity at excited state can also be rationalized by their excitation spectra (Figure S5), providing a more persuasive argument from another point of view. In general, the dual emission of fluorescence and p-RTP from HCE, PERTC, PERTB, and TBM at room temperature not only broadens new connotations for XB-relating researches, also provides strong fundamental evidence for proving the rationality and generality of the CTE mechanism.

To uncover more details of PL from these haloalkanes which are concealed by the weak luminescence due to the frequent molecular motions even under the restraints of lattice, cryogenic experiment was carried out to further rigidify the conformations. Upon cooling down to $77 \mathrm{~K}$, as shown in Figure 3A and S6, besides the extensively boosted intensity of their prompt and delayed emission, highly prolonged afterglows were also acquired, which is up to $8 \mathrm{~s}$ for PERTC and $12 \mathrm{~s}$ for PERTB crystals $\left(\lambda_{\mathrm{ex}}=312 \mathrm{~nm}\right.$, Figure S6), accompanying greatly extended $<\tau_{\tau}>_{\mathrm{p}}$ s values (Figure 3D, S7). As demonstrated in Figure 3B taking PERTC as the representative, compared with the main peaks in UV-A band at room temperature, visible portion of emission with peaks at $\sim 450 \mathrm{~nm}$ was considerably enhanced and became predominant, thus illustrating the increment of visible emission in PL photographs (Figure 3A). Transformation of main peaks and different increasing extents of UV-A and visible emission can be ascribed to the heterogenous population of emissive halogen clusters which have diverse responses to temperature change. 

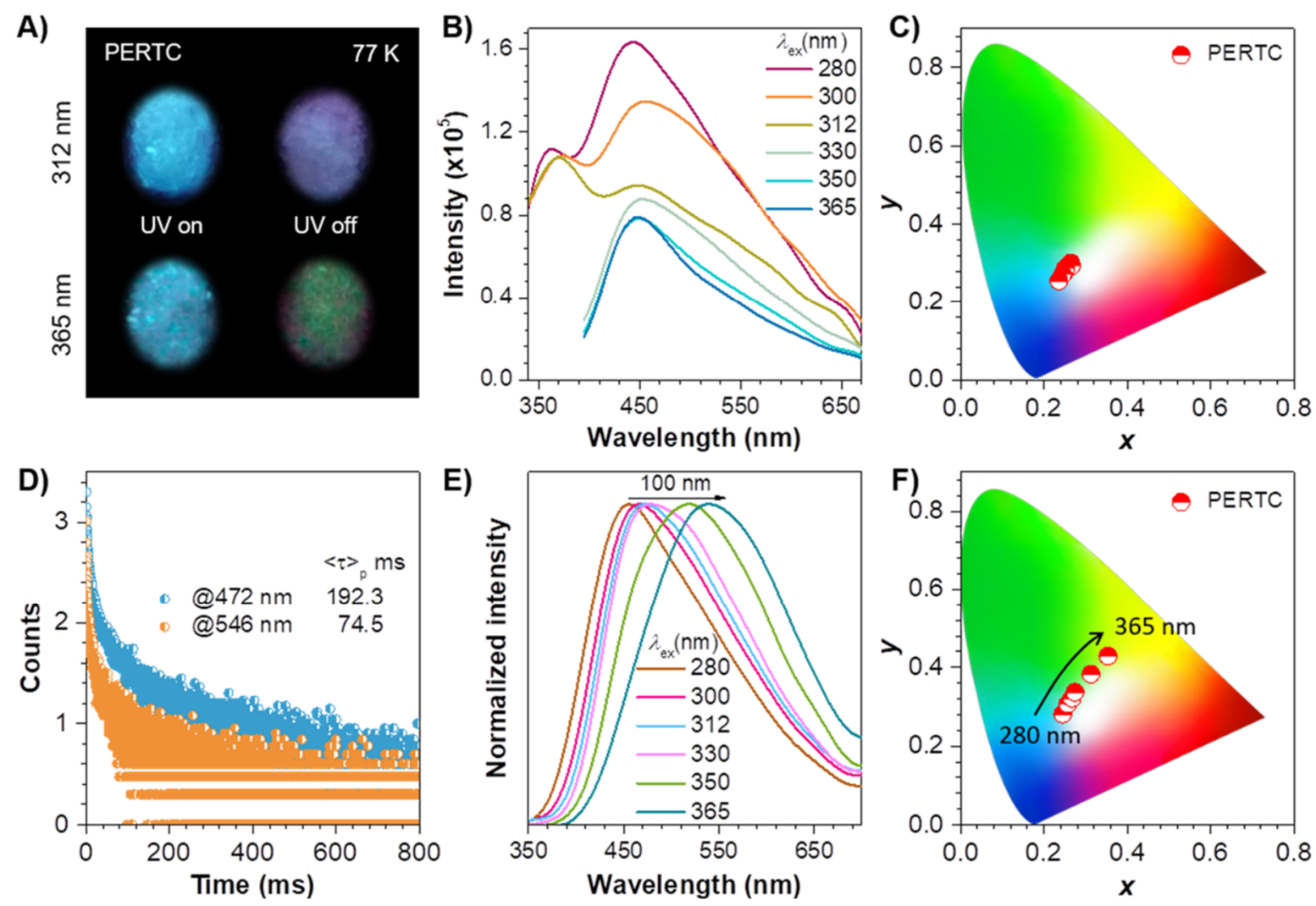

Figure 3. A) Luminescent photographs of PERTC single crystals taken under or after ceasing 312 and $365 \mathrm{~nm}$ UV irradiation at $77 \mathrm{~K} . \mathrm{B})$ Prompt $\left(t_{\mathrm{d}}=0\right)$ emission spectra and C) CIE coordinate diagram of PERTC crystals with different $\lambda_{\mathrm{ex}} \mathrm{s}$ at $77 \mathrm{~K}$. D) Ms scale time-resolved measurements of lifetimes for PERTC crystals monitored at varying $\lambda_{\mathrm{ems}}\left(\lambda_{\mathrm{ex}}=312 \mathrm{~nm}\right)$ at $77 \mathrm{~K}$. E) Delayed $\left(t_{\mathrm{d}}=0.1 \mathrm{~ms}\right)$ emission spectra and F) CIE coordinate diagram of PERTC crystals with different $\lambda_{\mathrm{ex}} \mathrm{S}$ at $77 \mathrm{~K}$.

Moreover, from PERTC crystals, $\lambda_{\text {ex }}$-dependent emission with slight trend of redshift and then blueshift as $\lambda_{\mathrm{ex}}$ increases was also observed (Figure 3B), which is confirmed by their corresponding Commission International de l'Éclairage (CIE) coordinates (Figure 3C). Notably, such $\lambda_{\text {ex }}$-dependence is much more striking in delayed emission, for which color-tunable phosphorescence is obtained. Variable peaks with broad full-width at half-maximum (FWHM) from blue to yellowish-green were observed with $\lambda_{\mathrm{ex}}$ changing from 280 to $365 \mathrm{~nm}$, with redshift for $\sim 100 \mathrm{~nm}$ (Figure 3E), which are consistent with the CIE coordinates from $(0.25,0.28)$ to $(0.36,0.43)$ (Figure $3 F)$. Furthermore, for other three haloalkane crystals, similar main peak transformation and $\lambda_{\text {ex }}$-dependent PL were also perceived (Figure S8-S10). Specifically, 
TBM crystals even exhibit broader region of phosphorescence tunability from dark blue to yellowishgreen (Figure S10C) with CIE coordinates varying from $(0.17,0.14)$ to $(0.33,0.42)$ (Figure S10D). Miscellaneous emissive halogen clusters are supposed to be stabilized under cryogenic circumstances, leading to immensely promoted and color-tunable PL, especially afterglows, in response to variable $\lambda_{\mathrm{ex}} \mathrm{S}$.

As diverse emissive species adopt various responses to temperature change and due to the better tunability of phosphorescence, delayed emission of these crystals at different temperatures were further studied. As expected, the phosphorescence of HCE shows apparent tunability in response to the ascending of temperature, which is firstly redshifted from 550 to $600 \mathrm{~nm}$ and then blueshifted to $560 \mathrm{~nm}$ (Figure 4A) together with the CIE coordinates varying from $(0.41,0.49)$ to $(0.46,0.46)$ and then to $(0.38,0.43)$ (Figure 4B). However, for the other three crystals, merely simple redshift trend with the increment of temperature is observed (Figure S11-S13). Furthermore, an extremely broad FWHM of up to $155 \mathrm{~nm}$ implies multiple emissive clusters in HCE crystals, whose characteristic peaks fluctuate with the variation in temperature (Figure S14) respectively. Plus, as Figure S14 exhibits, the distribution of main and shoulder peaks is disparate owing to discrepant responses to temperature from characteristic emission of these diverse emissive species, thus leading to different contributions to the outer luminescence and consequently generating temperature-dependent phosphorescence. It is also noted that as temperature rises, phosphorescence intensity of HCE crystals at $550 \mathrm{~nm}$ exhibits an obvious dual-linear phase with the intersection point at $218 \mathrm{~K}$ (Figure 4C). To acquire more information of that, differential scanning calorimetry (DSC) scans were performed, in which a subtle transition between endothermal and exothermal portion at $\sim 218 \mathrm{~K}$ was noticed (Figure 4D). Thus we can speculate that some sort of crystallographic transition may happen at such temperature and the temperature-dependent emission empowers it a feasible method to detect such transitions. 

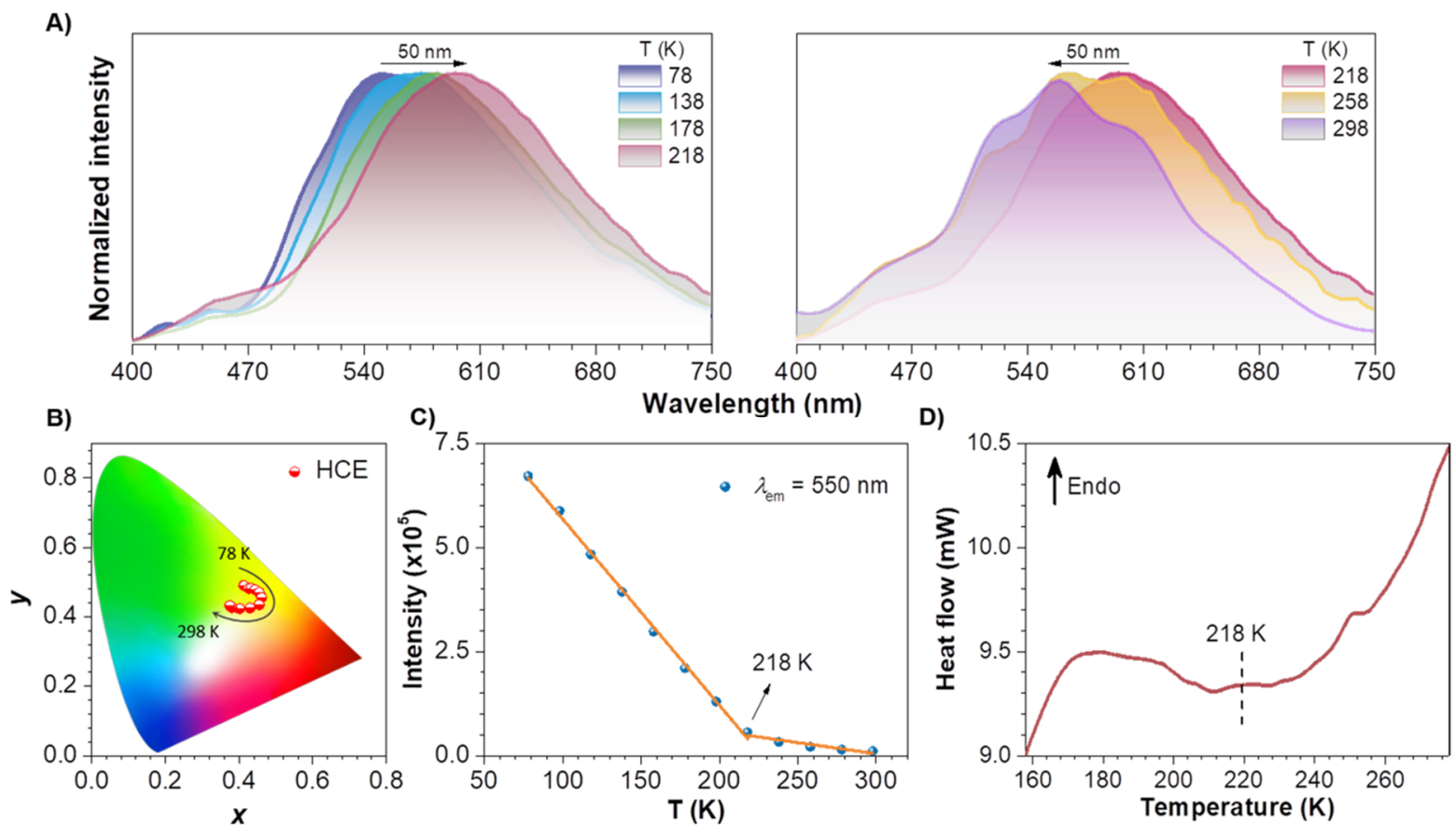

Figure 4. A) Delayed ( $\left.t_{\mathrm{d}}=0.1 \mathrm{~ms}\right)$ emission spectra and B) corresponding CIE coordinate diagram of HCE crystals at different temperatures $\left(\lambda_{\mathrm{ex}}=312 \mathrm{~nm}\right)$. The delayed emission spectra is divided into two parts to show the trend of redshift and blueshift. C) Linear fitting of luminescent intensity of HCE crystals at $550 \mathrm{~nm}\left(\lambda_{\mathrm{ex}}=312 \mathrm{~nm}\right)$. D) DSC curve of HCE crystals scanned at $100{ }^{\circ} \mathrm{C} / \mathrm{min}$.

When placed in vacuum, the PL, particularly p-RTP, from PERTB crystals can be improved (Figure S15). Nevertheless, the p-RTP was still too weak to be visualized. When compressed into tablets, PERTB, however, depicts remarkably boosted PL with vivid contrast (Figure 5A). Moreover, after removing irradiation, distinct green p-RTP is effectively visualized in PERTB tablets with prolonged $\langle\tau\rangle_{\mathrm{p}} \mathrm{s}$ (Figure 5E). Furthermore, upon pressurization (750 MPa), remarkably redshifted emission maximum (from 355 to $438 \mathrm{~nm}$ ) and narrowed FWHM (from 167 to $124 \mathrm{~nm}$ ) of PERTB are noticed (Figure 5B). Meanwhile, emerging new peaks (398/438/448 nm) are found (Figure 5B). Similar phenomena are also observed for PERTC (Figure 5C, 5D). For HCE and TBM, redshifted trend is also perceived in their prompt emission spectra (Figure S16). However, what varies is that the p-RTP of them is still too weak to be captured by digital cameras. For all these haloalkanes, although without conspicuous peak shifts in transient emission spectra, tablets are perceived to emit stronger phosphorescence at redder region than single crystals 
(Figure S17A-D), leading to the redshifted triplet emission (Figure S17E-H). These results should be attributed to the enhancement of short contacts and consequently TSCs among halogen atoms, which also induce simultaneously rigidified conformations. Such deduction was confirmed by the contrast of excitation spectra of their single crystals and tablets (Figure S18), where more extended peaks of tablets imply the presence of expanding conjugations. Thus, in summary, the multi-tunable PL in response to $\lambda_{\text {ex }}$, temperature, and pressure confirm the coexistence of miscellaneous emissive species in these haloalkanes from disparate aspects, offering new implications to the origin of PL and promising emerging applications of the compounds with halogen clusters.

A)
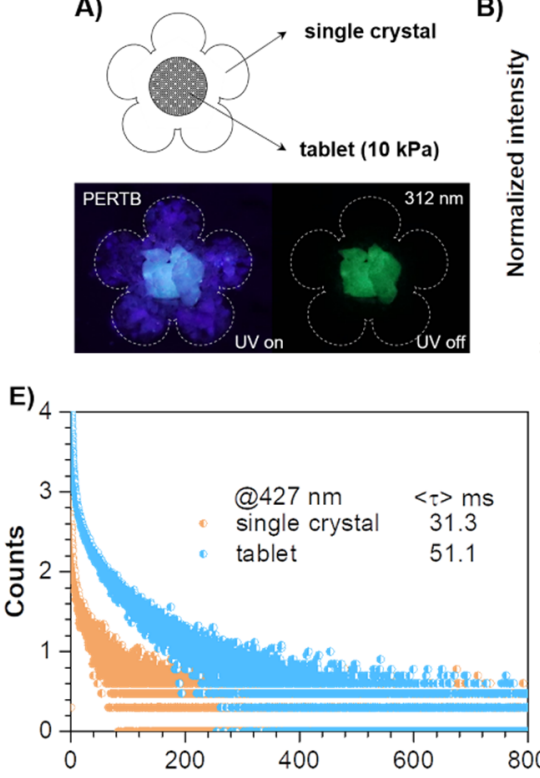

B)
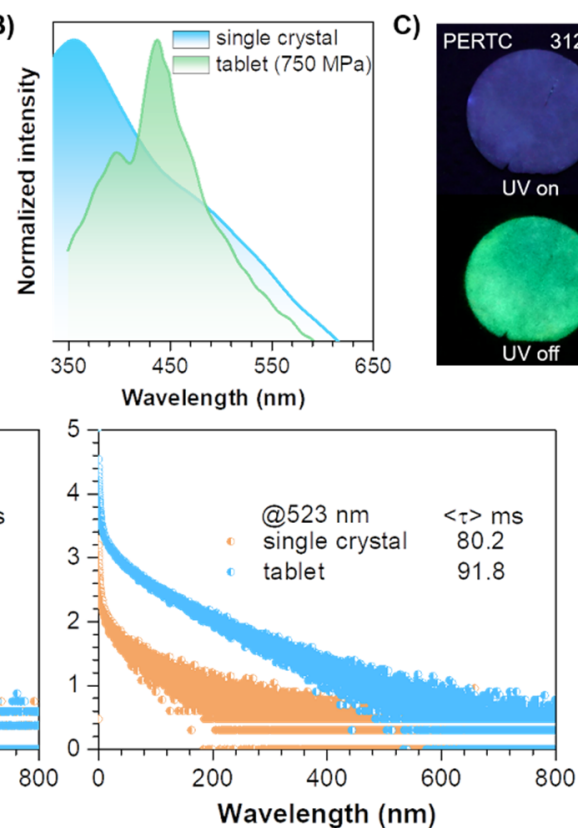

Wavelength $(\mathrm{nm})$
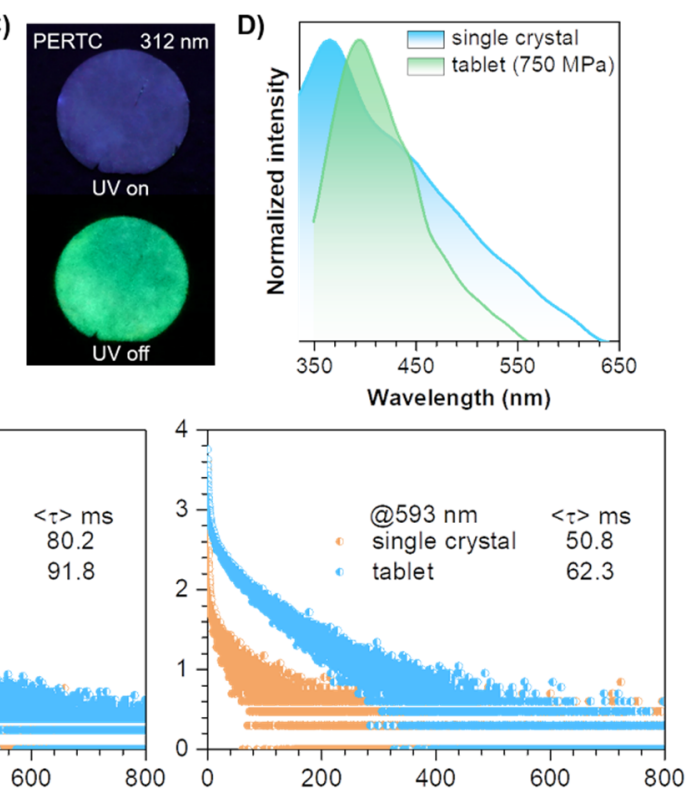

Figure 5. A) Illustration of PERTB a flower patten and corresponding luminescent photographs taken under or after ceasing the $312 \mathrm{~nm}$ UV irradiation. B) Prompt emission spectra of PERTB crystals and tablets. C) Luminescent photographs and D) prompt emission spectra of PERTC crystals and tablets taken under or after ceasing the $312 \mathrm{~nm}$ UV irradiation.

As demonstrated in Figure 6A, CTE mechanism can rationalize the dual emission and multi-tunable PL of these haloalkanes. Although isolated haloalkanes are not capable of emitting, extended conjugations and descending energy gaps of aggregated counterparts can lead to noticeable PL. Meanwhile, the 
concurrence of diverse emissive halogen clusters sensitive to temperature and pressure results in multitunable PL. To further illustrate the origin of PL from these haloalkanes, their single crystal structures were determined. However, due to extremely active molecular motions at room temperature, the preliminary single crystal determination was failed with no effective results acquired, so that such characterization can only be proceeded under cryogenic circumstance with the aim of restricting fierce molecular motions (Table S1). As shown in Figure 6B and S19, analyzed at $173 \mathrm{~K}$, abundant inter- and intramolecular $\mathrm{Cl} \cdots \mathrm{Cl}$ short contacts play essential roles in HCE molecules, which remarkably diminish the molecular motions, thus leading to restrained nonradiative dissipation and rigidified conformation. Also, thanks to electron-rich feature of $\mathrm{Cl}$ atoms and efficient interactions, 3D TSC network amongst multiple $\mathrm{n}$ electron-rich HCE molecules can be effectively facilitated. Therefore, although individual molecules are not qualified to allow efficient excitation and emission, aggregated molecules with halogen clusters are able to generate extensive electron communication and extended delocalizations, consequently bringing about diverse emissive clusters responsible for multi-tunable PL. Such multitunability thus can be well understood by the CTE mechanism. Furthermore, considering the presence of ample $\mathrm{n}$ electrons, together with the heavy atom effect of halogens, the phosphorescence emission can be rationalized by the strikingly promoted SOC and ISC process. Moreover, the interactions in TSC network and conformational rigidity would be strengthened under cryogenic circumstances, which could lead to brighter PL with better multi-tunability. For TBM, single crystal analysis has already been performed at $123 \mathrm{~K}$ by the previous publication, ${ }^{33}$ wherein effective TSC network based on multiple $\mathrm{Br} \cdots \mathrm{Br} \mathrm{XBs}$ is noticed (Figure S20). On the other hand, although severe disorder was found in PERTC and PERTB during their single crystal analyses even at $173 \mathrm{~K}$ (Figure S21) due to their extremely active molecular motions, ${ }^{39}$ akin structural features concluded from HCE and TBM can still be deduced from them. 


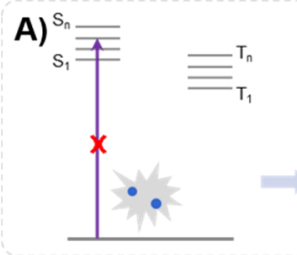

C)

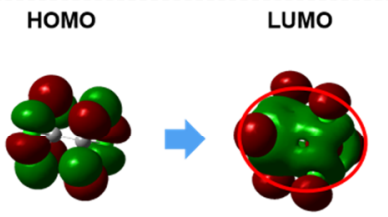

Monomer

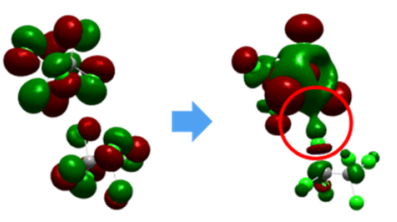

Dimer
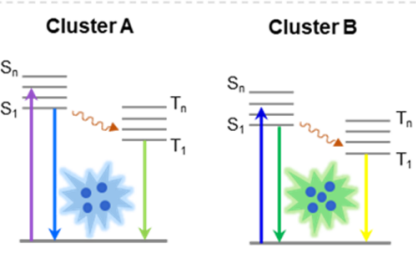

HOMO

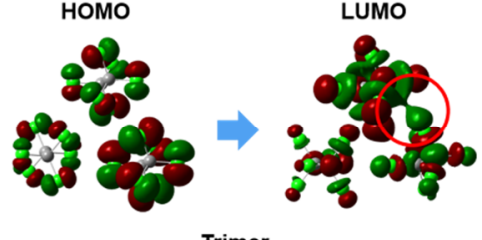

Trimer

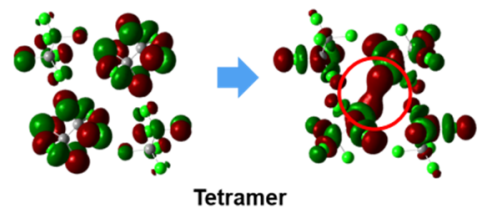

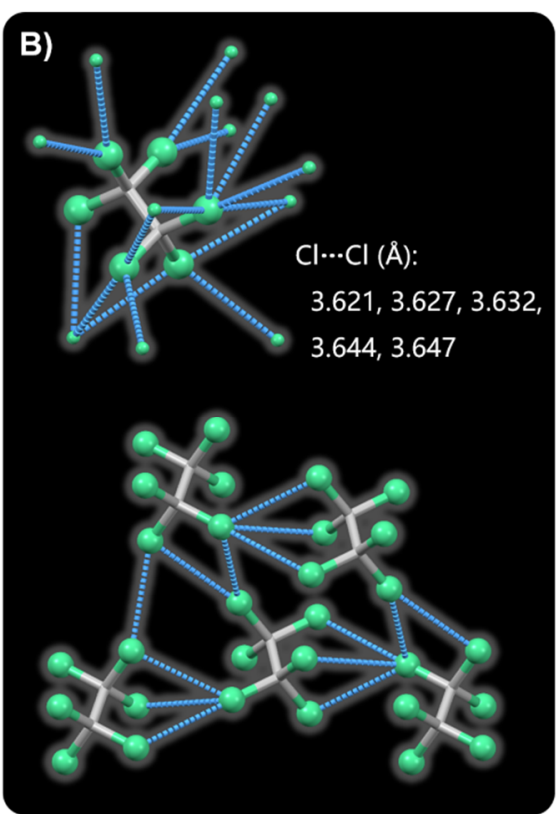

Figure 6. A) Jablonski diagrams for the generation of tunable PL. B) Crystal structure (173 K) with denoted intermolecular interactions around one molecule and fragmental molecular packing with TSC for HCE. C) Electron densities of the HOMO and LUMO levels for monomer, dimer, trimer and tetramer of HCE.

To acquire further information into the mechanism, theoretical calculations for monomer, dimer, trimer, and tetramer of HCE and TBM were conducted, which again support these judgements by demonstrating efficient intra- and intermolecular TSC. As depicted in Figure 6C, all HOMOs are mainly distributed on $\mathrm{Cl}$ atoms in the molecules without any overlap with one another, whereas conspicuous TSC among LUMOs, from monomer to tetramer, are observed, corresponding to the excited states. By forming multifarious emissive halogen clusters, such extensive TSC effectively narrows the energy gap between the ground and excited states and provides ample triplet energy levels (Figure 6C, S22 and Table S2), thus giving rise to the unique multi-tunable luminescence. Similarly, theoretical results for TBM also reveal effective TSCs and reduced energy gaps owing to the formation of Br clusters (Figure S23, S24 and Table S3). These results are consistent with the CTE mechanism and well rationalized the origin of the multi-tunable PL of the haloalkanes. 
To conclude, to the best of our knowledge, first examples of emissive halogen clusters were demonstrated. Photophysical properties of representative crystals of haloalkanes, namely HCE, PERTC, PERTB, and TBM were investigated at ambient conditions and $77 \mathrm{~K}$. Impressive fluorescencephosphorescence dual emission from the crystals were observed, on account of the formation of halogen clusters with effective TSCs and stiffened conformations. Furthermore, single crystal analyses and theoretical calculations clearly demonstrate the essential role played by $\mathrm{X} \cdots \mathrm{X}$ short contacts and consequent TSC network of halogen clusters in facilitating effective electron communication, conformational rigidity, and PL tunability that is responsive to multiple factors such as excitation wavelength, temperature and pressure. These results not only disclose the emissive capacity of halogen clusters, but also testify the rationality of the CTE mechanism for nonconventional luminophores. It is anticipated that this research could prompt exploration toward fundamental understanding of mechanism of nonconventional luminophores and shed new lights on exploiting potential applications of halogen involved electronic communications.

\section{Acknowledgement}

This work was sponsored by the National Natural Science Foundation of China $(51822303,52073172)$ and the Natural Science Foundation of Shanghai (20ZR1429400).

\section{Reference}

1. Legon, A. C. Angew. Chem. Int. Ed. 1999, 38, 2686.

2. Cavallo, G.; Metrangolo, P.; Milani, R.; Pilati, T.; Priimagi, A.; Resnati, G.; Terraneo, G. Chem. Rev. 2016, 116, 2478.

3. Shen, Q. J.; Jin, W. J. Phys. Chem. Chem. Phys. 2011, 13, 13721.

4. Clark, T.; Hennemann, M.; Murray, J. S.; Politzer, P. J. Mol. Model. 2007, 13, 291.

5. Erdélyi, M. Chem. Soc. Rev. 2012, 41, 3547. 
6. Mukherjee, A.; Sanz-Matias, A.; Velpula, G.; Waghray, D.; Ivasenko, O.; Bilbao, N.; Harvey, J. N.; Mali, K. S.; De Feyter, S. Chem. Sci. 2019, 10, 3881.

7. Foyle, É. M.; White, N. G. CrystEngComm 2020, 22, 2526.

8. Xing, L.; Jiang, W.; Huang, Z.; Liu, J.; Song, H.; Zhao, W.; Dai, J.; Zhu, H.; Wang, Z.; Weiss, P. S. Chem. Mater. 2019, 31, 3041.

9. Ungati, H.; Govindaraj, V.; Mugesh, G. Angew. Chem. Int. Ed. 2018, 57, 8989.

10. Caballero, A.; Zapata, F.; White, N. G.; Costa, P. J.; Félix, V.; Beer, P. D. Angew. Chem. Int. Ed. 2012, 51, 1876.

11. Meazza, L.; Foster, J. A.; Fucke, K.; Metrangolo, P.; Resnati, G.; Steed, J. W. Nat. Chem. 2013, 5, 42.

12. Metrangolo, P.; Neukirch, H.; Pilati, T.; Resnati, G. Acc. Chem. Res. 2005, 38, 386.

13. Yang, H.; Li, M.; Li, C.; Luo, Q.; Zhu, M. Q.; Tian, H.; Zhu, W. H. Angew. Chem. Int. Ed. 2020, 59, 8560.

14. Yuan, Y.; Xu, S.; Cheng, X.; Cai, X.; Liu, B. Angew. Chem. Int. Ed. 2016, 55, 6457.

15. Li, Y.; Xu, Z.; Zhu, X.; Chen, B.; Wang, Z.; Xiao, B.; Lam, J. W.; Zhao, Z.; Ma, D.; Tang, B. Z. ACS Appl. Mater. Interfaces 2019, 11, 17592.

16. Zhang, Q.; Li, J.; Shizu, K.; Huang, S.; Hirata, S.; Miyazaki, H.; Adachi, C. J. Am. Chem. Soc. 2012, $134,14706$.

17. Tao, Y.; Yuan, K.; Chen, T.; Xu, P.; Li, H.; Chen, R.; Zheng, C.; Zhang, L.; Huang, W. Adv. Mater. 2014, 26, 7931.

18. Wang, C.; Yu, Y.; Yuan, Y.; Ren, C.; Liao, Q.; Wang, J.; Chai, Z.; Li, Q.; Li, Z. Matter 2020, 2, 181.

19. Xu, S.; Duan, Y.; Liu, B. Adv. Mater. 2020, 32, 1903530.

20. Zhou, Y.; Qin, W.; Du, C.; Gao, H.; Zhu, F.; Liang, G. Angew. Chem. Int. Ed. 2019, 58, 12102.

21. Chen, C.; Ni, X.; Jia, S.; Liang, Y.; Wu, X.; Kong, D.; Ding, D. Adv. Mater. 2019, 31, 1904914. 
22. Wang, X.; Li, M.; Hou, Y.; Li, Y.; Yao, X.; Xue, C.; Fei, Y.; Xiang, Y.; Cai, K.; Zhao, Y.; Luo, Z. Adv. Funct. Mater. 2020, 30, 2000229.

23. Sivchik, V. V.; Solomatina, A. I.; Chen, Y. T.; Karttunen, A. J.; Tunik, S. P.; Chou, P. T.; Koshevoy, I. O. Angew. Chem. Int. Ed. 2015, 54, 14057.

24. Cai, S.; Shi, H.; Tian, D.; Ma, H.; Cheng, Z.; Wu, Q.; Gu, M.; Huang, L.; An, Z.; Peng, Q. Adv. Funct. Mater. 2018, 28, 1705045.

25. Yan, D.; Delori, A.; Lloyd, G. O.; Friščić, T.; Day, G. M.; Jones, W.; Lu, J.; Wei, M.; Evans, D. G.; Duan, X. Angew. Chem. Int. Ed. 2011, 50, 12483.

26. Bolton, O.; Lee, K.; Kim, H.-J.; Lin, K. Y.; Kim, J. Nat. Chem. 2011, 3, 205.

27. Shimizu, M.; Kimura, A.; Sakaguchi, H. Eur. J. Org. Chem. 2016, 2016, 467.

28. Wang, W.; Zhang, Y.; Jin, W. J. Coord. Chem. Rev. 2020, 404, 213107.

29. Ding, X. H.; Chang, Y. Z.; Ou, C. J.; Lin, J. Y.; Xie, L. H.; Huang, W. Natl. Sci. Rev. 2020, DOI: 10.1093/nsr/nwaa170.

30. Liu, B.; Zhang, H.; Liu, S.; Sun, J.; Zhang, X.; Tang, B. Z. Mater. Horiz. 2020, 7, 987.

31. Zheng, S.; Zhu, T.; Wang, Y.; Yang, T.; Yuan, W. Z. Angew. Chem. Int. Ed. 2020, 59, 10018.

32. Zhou, Q.; Yang, T.; Zhong, Z.; Kausar, F.; Wang, Z.; Zhang, Y.; Yuan, W. Z. Chem. Sci. 2020, 11, 2926.

33. Lindeman, S. V.; Hecht, J.; Kochi, J. K. J. Am. Chem. Soc. 2003, 125, 11597.

34. Gong, Y.; Tan, Y.; Mei, J.; Zhang, Y.; Yuan, W.; Zhang, Y.; Sun, J.; Tang, B. Z. Sci. China. Chem. 2013, 56, 1178.

35. Zhou, Q.; Cao, B.; Zhu, C.; Xu, S.; Gong, Y.; Yuan, W. Z.; Zhang, Y. Small 2016, 12, 6586.

36. Tomalia, D. A.; Klajnert-Maculewicz, B.; Johnson, K. A.-M.; Brinkman, H. F.; Janaszewska, A.; Hedstrand, D. M. Prog. Polym. Sci. 2019, 90, 35.

37. Shang, C.; Zhao, Y.; Long, J.; Ji, Y.; Wang, H. J. Mater. Chem. C 2020, 8, 1017.

38. Feng, Y.; Bai, T.; Yan, H.; Ding, F.; Bai, L.; Feng, W. Macromolecules 2019, 52, 3075. 
39. Klaeboe, P.; Klewe, B.; Martinsen, K.; Nielsen, C.; Powell, D.; Stubbles, D. J. Mol. Struct. 1986, 140,1 .

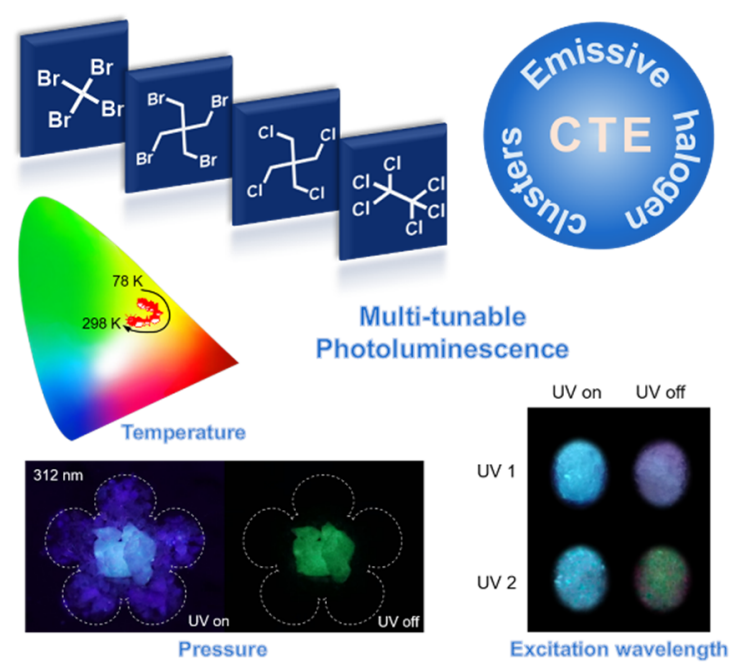

Emissive halogen clusters: By forming multitudinous halogen-halogen short contacts, the first examples of halogen clusters that are fluorescent-phosphorescence dual emissive were reported. Furthermore, the luminescence is readily tunable in response to excitation wavelength, temperature, and pressure. These results uncover the new feature of halogen clusters and provide strong implications for the emission mechanism of nonconventional luminophores. 